\title{
Edizioni di riferimento e abbreviazioni
}

Le opere di Dante, salvo diversa indicazione, sono citate dalle seguenti edizioni:

- La Commedia secondo l'antica vulgata, a cura di G. Petrocchi, Milano 19661967 (rist. Firenze 1994).

- Convivio, a cura di F. Brambilla Ageno, Firenze 1995.

- De Vulgari Eloquentia, a cura di P. V. Mengaldo, Padova 1968.

- Epistole, a cura di M. Baglio, in Nuova Edizione commentata delle Opere di Dante, V, Epistole Egloge Questio de aqua et terra, a cura di M. Baglio, L. Azzetta, M. Rinaldi, Roma 2016, pp. 3-269.

- Epistola XIII, a cura di L. Azzetta, in Nuova Edizione commentata delle Opere di Dante, V, Epistole Egloge Questio de aqua et terra, a cura di M. Baglio, L. Azzetta, M. Petoletti, M. Rinaldi, Roma 2016, pp. 273-287.

- Rime, a cura di D. De Robertis, Firenze 2002.

- La Vita Nova, a cura di G. Gorni, in Opere, vol. I. Rime, Vita Nova, De vulgari eloquentia, Milano 2011.

Per quanto riguarda l'apparato di commento, le diverse edizioni delle opere di Dante sono citate attraverso le seguenti abbreviazioni:

Canz. (Allegretti) = Dante Alighieri, La canzone "montanina", Verbania 2001.

Comm (Tommaseo $)=$ Dante Alighieri, Divina Commedia, a cura di N. Tommaseo, Milano 1869.

Comm. (Inglese) = D. Alighieri, Commedia . Opera completa, revisione del testo e commento di G. Inglese, Roma 2016.

Conv. (Fioravanti) $=$ D. Alighieri, Convivio, a cura di G. Fioravanti, in Opere, vol. II. Convivio, Monarchia, Epistole, Egloge, Milano 2014.

Dve $($ Fenzi $)=$ D. Alighieri, De vulgari eloquentia, a cura di E. Fenzi, in Nuova edizione Commentata delle Opere di Dante, III, Roma 2012.

Dve $($ Marigo $)=$ D. Alighieri, De vulgari eloquentia, ridotto a miglior lezione, commentato e tradotto da A. Marigo, Firenze 1957.

Dve $($ Mengaldo $)=$ D. Alighieri, De vulgari eloquentia, a cura di P. V. Mengaldo, in Opere minori, II, Milano-Napoli 1979.

Dve $($ Tavoni $)=$ D. Alighieri, De vulgari eloquentia, a cura di M. Tavoni, in Opere, I. Rime, Vita Nova, De vulgari eloquentia, Milano 2011.

Egl. (Petoletti) = Dante Alighieri, Egloge, a cura di M. Petoletti, in Nuova Edizione commentata delle Opere di Dante, V, Epistole Egloge Questio de aqua et terra, a cura di M. Baglio, L. Azzetta, M. Petoletti, M. Rinaldi, Roma 2016.

Ep. $($ Del Monte) $=$ D. Alighieri, Epistole, a cura di A. Del Monte, in Opere minori, Milano 1960. 
Ep. $($ Fraticelli) $=$ Il Convito di D. Alighieri e le epistole, con illustrazioni e note di P. Fraticelli e d'altri, Firenze 1857.

Ep. $\left(\right.$ Fraticelli $\left.{ }^{2}\right)=$ Il Convito di D. Alighieri e le epistole, con illustrazioni e note di P. Fraticelli e d'altri, II ed., Firenze 1862.

Ep. (Brugnoli-Frugoni) = D. Alighieri, Epistole, a cura di G. Brugnoli, A. Frugoni, in Opere minori, tomo II, Milano-Napoli 1979.

Ep. (Giuliani) = Le opere latine di Dante Allighieri, reintegrate nel testo con nuovi commenti da G. Giuliani, vol. II. Epistolae, Eclogae e Quaestio de aqua et terra, Firenze 1882.

Ep. (Honess) = D. Alighieri, Four political letters, transl. and with a commentary by C. E. Honess, London 2007.

Ep. (Jacomuzzi) = D. Alighieri, Epistole, a cura di A. Jacomuzzi, in Opere minori, II, Torino 1986.

Ep. $($ Lokaj $)=$ D. Alighieri, Epistole, a cura di R. Lokaj, in Opere latine, Roma 2005.

Ep. $($ Monti $)=$ A. Monti, Le lettere di Dante. Testo, versione, commento e appendici, Milano 1921.

Ep. $($ Moore $)=$ D. Alighieri, Epistole, in Tutte le opere di D. Alighieri, nuovamente rivedute nel testo dal Dr. E. Moore, Oxford 1894 (rist. 1897, 1904).

Ep. (Pastore Stocchi) = D. Alighieri, Epistole, Ecloge, Questio de situ et forma aque et terre, a cura di M. Pastore Stocchi, Roma-Padova 2012.

Ep. $($ Pézard $)=$ D. Alighieri, Epîtres, in Oeuvres complètes, éd. par A. Pézard, Paris 1965.

Ep. $($ Pistelli $)=$ D. Alighieri, Epistole, in Le Opere di Dante. Testo critico della Società Dantesca Italiana, Firenze 1921 (rist. 1960).

Ep. (Torri) = Epistole di Dante Allighieri edite e inedite. Aggiuntavi la dissertazione intorno all'acqua e alla terra e le traduzioni rispettive a riscontro del testo latino con illustrazioni e note di diversi, a cura di A. Torri, Livorno 1842.

Ep. (Toynbee) = Dantis Alagherii Epistolae. The Letters of Dante, Emended Text, with Introduction, Translation, Notes, and Indices and Appendix on the Cursus by P. Toynbee, Oxford 1920.

Ep. (Mazzoni) = D. Alighieri, Epistole I-V. Saggio di edizione critica, a cura di F. Mazzoni, Milano 1967.

Ep. $($ Villa $)$ = D. Alighieri, Epistole, in Opere, II, Convivio, Monarchia, Epistole, Egloge, Milano 2014.

Ep. (Vinay) = D. Alighieri, Monarchia, testo, introduzione e commento a cura di G. Vinay, in appendice Le Epistole politiche tradotte, Firenze 1950.

Ep. $($ Witte $)=$ Dantis Alligherii Epistolae quae exstant cum notis Caroli Witte ..., Pavia 1827.

Inf. (Bellomo) = D. Alighieri, Inferno, a cura di S. Bellomo, Torino 2013. 
Mon. (Chiesa-Tabarroni) = D. Alighieri, Monarchia, a cura di P. Chiesa, A. Tabarroni, in Nuova Edizione Commentatata delle Opere di Dante, IV, Roma 2013. Mon. (Quaglioni) = D. Alighieri, Monarchia, a cura di D. Quaglioni, in Opere, II. Convivio, Monarchia, Epistole, Egloge, Milano 2014.

Mon. (Ricci) = Dante Alighieri, Monarchia, a cura di P. G. Ricci, Milano 1965.

Mon. (Shaw) = Dante Alighieri, Monarchia, a cura di P. Shaw, Firenze 2009.

Op. minori (Fraticelli) = Dantis Aligherii Epistolae quae extant, cum disquisitionibus atque italica interpretatione Petri Fraticelli, Firenze 1840.

Rime (De Robertis) = Dante Alighieri, Rime, a cura di D. De Robertis, Firenze 2002.

Rime (Witte) = D. Alighieri, Lyrische Gedichte, ed. Karl Witte, Lipzig 1842.

Volgarizzamento (Montefusco) = Appendice III. I Volgarizzamenti delle epistole $V$ e VII, in Nuova Edizione Commentata delle Opere di Dante, V, Epistole Egloge Quaestio de aqua et terra, a cura di M. Baglio, L. Azzetta, M. Petoletti, M. Rinaldi, Roma 2016.

Altre abbreviazioni:

CDD = Codice Diplomatico Dantesco, a cura di T. De Robertis, G. Milani, L. Regnicoli e S. Zamponi, in Nuova Edizione commentata delle Opere di Dante, VII, III, Roma 2016.

CSEL = Corpus Scriptorum Ecclesiasticorum Latinorum, Vienna 1866-.

DBI = Dizionario Biografico degli Italiani, Roma 1960-.

ED = Enciclopedia Dantesca, Roma 1970-1978.

MGH = Monumenta Germaniae Historica.

MGH, SS = Monumenta Germaniae Historica, Scriptores.

MGH, LL = Monumenta Germaniae Historica, Leges.

$\mathrm{MGH}, \mathrm{EE}=$ Monumenta Germaniae Historica, Epistolae.

NECOD = Nuova edizione commentata delle opere di Dante, Roma 2012-.

$\mathrm{PL} \quad=$ Patrologiae Cursus completus [...] Series latina [...], a cura di J. P. Migne, Paris 1844-1864.

RIS $=$ Rerum italicarum scriptores, Milano 1723-1751.

RIS ${ }^{2}=$ Rerum italicarum scriptores, Città di Castello 1900-1975. 
